\title{
Induction of macrocyst wall formation by ZYG1 in Dictyostelium discoideum
}

\author{
Aiko Amagai ${ }^{*}$, Fumio Takahashi ${ }^{1,2,3}$, Toshinori Usui ${ }^{4,5}$, Tomoaki Abe ${ }^{4}$ and Yasuo Maeda ${ }^{1}$ \\ *Correspondence: aiamagai@amber.plala.or.jp \\ 'Graduate School of Life Sciences, Tohoku University, Sendai 980-8577, Japan. \\ ${ }^{2}$ PRESTO, Japan Science and Technology Agency, Kawaguchi, Saitama 332-0012, Japan. \\ ${ }^{3}$ Department of Biotechnology, College of Life Sciences, Ritsumeikan University, Nojihigashi, Kusatsu, Shiga 525-8577, Japan. \\ ${ }^{4}$ Department of Biological Sciences, Faculty of Science and Engineering, Ishinomaki Senshu University, Ishinomaki, Miyagi 986-0031, \\ Japan. \\ ${ }^{5}$ Tohoku Medical Megabank Organization, 2-1 Seiryo-Machi Aoba-ku, Sendai 980-8574, Miyagi, Japan.
}

\begin{abstract}
Background: Macrocyst is known as the sexual developmental form in cellular slime molds. ZYG1 is known to be involved in zygote formation by cell fusion during macrocyst formation in Dictyostelium mucoroides, one of the species of cellular slime molds.

Aim and methods: To know the effects of ZYG1 on the asexual and sexual development in Dictyostelium discoideum, an another species of cellular slime molds, we observed the developmental process and forms of a transformant $\left(\mathrm{GFP}-\mathrm{ZYG}_{1}{ }^{\mathrm{OE}}\right)$ derived from Dictyostelium discoideum, in which GFP-ZYG1 fusion protein is overproduced.

Results: GFP-ZYG1 ${ }^{\mathrm{OE}}$ cells were developed without or with an opposite mating type, V12M2 cells. When GFP-ZYG ${ }^{\mathrm{OE}}$ cells were developed without V12M2 cells, they formed macrocyst-like structures consisting of a central cell mass and a thick transparent macrocyst wall surrounding it. It was suggested that transparent zones in the macrocyst wall were formed by cell fusion. When GFP-ZYG ${ }^{\text {OE }}$ cells were developed with V12M2 cells, globular bodies which are likely the macrocysts surrounded by thin cellulosic walls were found besides macrocysts. They were formed directly from zygotic giant cells without the formation of cell aggregates. The formation of the globular body is not caused by ZYG1 overproduction, because GFP ${ }^{\mathrm{CONT}}$ cells, a transformant overproducing GFP protein as controls, also formed globular bodies. Conclusion: By the use of GFP-ZYG ${ }^{\text {OE }}$ cells overproducing GFP-ZYG1 fusion protein, it is revealed that ZYG1 is closely involved in macrocyst wall formation. This is the new finding concerning the function of ZYG1. In addition, the formation of a globular body, which is likely the macrocyst surrounded by a thin cellulosic wall, is demonstrated as a novel process of sexual development.
\end{abstract}

Keywords: Dictyostelium, cellulose, cell fusion, macrocyst wall, ZYG1

\section{Introduction}

Macrocysts are formed as a sexual developmental process in cellular slime molds. Removal of any growing medium (starvation) causes cells to stop growing and form cell aggregates. Macrocyst formation in Dm7, a wild-type strain of Dictyostelium mucoroides is characterized by the formation of large aggregates after starvation which are then subdivided into smaller masses (precysts), each of which is surrounded by a fibrillar sheath. A giant cytophagic cell arises at the center of each precyst, which engulfs all the other cells in the precyst. The engulfed cells (endocytes) become oval and are eventually broken down into granular remnants. The enlarged giant cell is eventually surrounded by a thick macrocyst wall to form a mature macrocyst. The macrocyst wall has been shown electron-microscopically to consist of three layers: a thin inner layer of the least electron-density, a thick middle layer of medium electron-density, and a thin electrondense outer layer [1]. The macrocyst wall is considered to be a product synthesized by a giant cell between the cell membrane 
Amagai et al. Research Journal of Developmental Biology 2014,

and the outer-most fibrillar sheath [1]. After a resting period, the macrocyst germinates to release several amoeboid cells and initiates a new life cycle [2].

There are mainly two kinds of mating systems involved in macrocyst formation: homothallic and heterothallic [3-6]. Dm7 is a homothallic strain, which forms macrocysts without mating types. Ax2, an axenic strain of Dictyostelium discoideum (D. discoideum) is a heterothallic strain, which mates with an opposite mating type strain, $\mathrm{V} 12 \mathrm{M} 2$. In the developmental process of macrocyst formation in Ax2 cells co-cultured with V12M2 cells, zygotic giant cells attract local amoebae, resulting in the formation of precysts $[7,8]$. Thus giant cells appear within aggregates during macrocyst formation in Dm7 cells [1], while the giant cells are formed before distinct cell aggregation in the case of Ax2 cells co-cultured with V12M2 cells. The process of macrocyst formation, however, is believed to be basically the same between the two mating systems $[9,10]$.

The cytophagic giant cells formed during macrocyst formation are zygotes produced by cell fusion and subsequent nuclear fusion [11]. As regulators of zygote formation, ethylene (a potent plant hormone) and 3', 5'-cyclic adenosine monophosphate (cAMP) have been identified. Ethylene induces zygote formation [11], while CAMP inhibits zygote formation $[12,13]$.

Concerning the signal transductions involved in zygote formation, the signaling pathway via $\mathrm{Ca}^{2+}$ and protein kinase $\mathrm{C}$ $(P K C)$ is suggested in both homothallic Dm7 and heterothallic Ax2 cells [13,14-18].

As a gene involved in zygote formation, zyg 1 has been isolated from a cDNA library of Dm7 by time dependent differential screening (DDBJ/EMBL/GenBank, accession number AB006956) and from a genomic DNA of Dm7 by PCR (DDBJ/EMBL/GenBank, accession number AB479506). Zyg 1

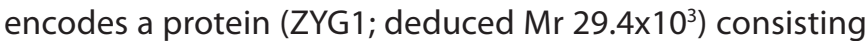
of 268 amino acids. According to BLAST and FASTA searches, ZYG1 is predicted to have PKC-mediated phosphorylation sites, but neither transmembrane domains nor specific signal sequences $[19,20]$. In a transformant over-expressing zyg 1 , the formation of zygotic giant cells is greatly enhanced, and macrocysts are formed even under unfavorable conditions [21]. Since the zyg 1 expression is enhanced by ethylene, ethylene induces zygote formation through an enhanced expression of zyg1 [22].

From the prediction that ZYG1 (a zyg1 product) has PKCmediated phosphorylation sites, ZYG1 is expected to be a candidate of a substrate for PKC. Using Ax2, instead of Dm7, this has been made clear. A transformant, GFP-ZYG1 ${ }^{\circ \mathrm{E}}$ (which is named as GFP/ZYG $1^{\mathrm{OE}}$ in [23]) derived from $A \times 2$, which overproduces GFP-ZYG1 fusion protein, is constructed. For this, a zyg 1 isolated from Dm7 is used, because the zyg 1 has not been found in D. discoideum by the Japanese Dictyostelium cDNA project and genome project of Dictyostelium [24]. Using GFP-ZYG1 ${ }^{\text {OE }}$ cells, it has been shown that ZYG1 is actually phosphorylated by PKC [23]. GFP-ZYG1 fusion protein uniformly distributes in the cytoplasm of GFP-ZYG1 ${ }^{\text {OE }}$ cells. In addition,
ZYG1 is translocated at regions of cell-to-cell contact where cell fusion occurs [23]. These results indicate that ZYG1 moves from the cytoplasm to the cell membrane, is phosphorylated by PKC and causes cell fusion through PKC mediated signal transduction pathways. Thus ZYG1 plays an important role in zygotic cell fusion.

The developmental process and forms of GFP-ZYG $1^{\text {OE }}$ cells co-cultured without or with an opposite mating type, V12M2 cells, were also examined during the course of the study. We report here a new function of ZYG1 and the unique process for macrocyst formation obtained consequently by use of GFP-ZYG ${ }^{\mathrm{OE}}$ derived from $A \times 2$ cells, which overproduces GFP-ZYG1 fusion protein.

\section{Materials and methods}

\section{Cell cultures and developmental conditions}

GFPCONT and GFP-ZYG $1^{\mathrm{OE}}$, transformants derived from Ax2 (an axenic strain of $D$. discoideum) (Amagai et al., 2012) and V12M2 (an opposite mating type strain of $A \times 2$ ) were used in this work. Vegetative GFPCONT and GFP-ZYG ${ }^{\mathrm{OE}}$ cells were grown axenically by shake cultures in PS-medium ( $1 \%$ Special Peptone [Oxoid: Lot No. 333 56412], 0.7\% Yeast extract (Oxoid), 1.5\% D-glucose, $0.11 \% \mathrm{KH}_{2} \mathrm{PO}_{4^{\prime}} 0.05 \% \mathrm{Na}_{2} \mathrm{HPO}_{4} \cdot 12 \mathrm{H}_{2} \mathrm{O}, 40 \mathrm{ng} / \mathrm{ml}$ vitamin $\mathrm{B}_{12^{\prime}}$ and $80 \mathrm{ng} / \mathrm{ml}$ folic acid) containing $200 \mu \mathrm{g} / \mathrm{ml}$ streptomycin, $10 \mu \mathrm{g} / \mathrm{ml}$ tetracycline and $50 \mu \mathrm{g} / \mathrm{ml}$ Geneticin (G418) (GIBCO $B R L$ ). To allow the cells to differentiate, they were harvested during the exponential growth phase, washed once in BSS (Bonner's salt solution: $10 \mathrm{mM} \mathrm{NaCl}, 10 \mathrm{mM} \mathrm{KCl}, 2.7 \mathrm{mM}$ $\left(\mathrm{CaCl}_{2}\right)$ [25] as a starvation medium. $\mathrm{V} 12 \mathrm{M} 2$ cells were grown by simultaneous culture with Escherichia coli B/r. Vegetative cells at the exponential growth phase were harvested and washed three times with BSS for starvation. Cell culture and development were carried out in dark conditions at $22^{\circ} \mathrm{C}$. For observation and time-lapse imaging under an inverted microscope (Nikon), the cells were settled on glass based dishes (Iwaki, 3910-035, 35 mm diameter) and developed under submerged conditions in BSS at $1 \sim 6 \times 10^{6} \mathrm{cells} / \mathrm{cm}^{2}$.

\section{Calcofluor staining}

Immediately after one drop of Calcofluor $(10 \mu \mathrm{g} / \mathrm{ml}$; Sigma $\mathrm{F}$-3397) was added into the $2 \mathrm{ml}$ cell suspension, one drop of cell suspension was placed on cleaned coverslips for observation.

\section{Imaging of living cells}

Glass dishes with adhered living cells were placed on an inverted microscope for observation (Nikon, Japan). Timelapsed images were acquired at one frame per 20 seconds using a CCD camera (Leica Dc250, Germany).

\section{Observations and photographs}

Developing cells and forms were observed and photographed under an inverted microscope, a phase-contrast microscope (Nikon, Tokyo) and a fluorescence microscope using UV excitation 
(Olympus, Tokyo).

\section{Results}

GFP-ZYG $1^{\mathrm{OE}}$ cells construct macrocyst-like structures without cells of the opposite mating type

The GFP-ZYG1 ${ }^{\text {OE }}$ cells and GFPCONT cells, transformants derived from Ax2 cells, are confirmed by western blot to overproduce GFP- ZYG1 fusion protein and GFP protein, respectively, at least during early development [23].

When GFP-ZYG $1^{\mathrm{OE}}$ cells were developed without V12M2 cells, GFP-ZYG $1^{\mathrm{OE}}$ cells formed loose aggregates without sheath materials at 1 day of development after starvation (Figure 1a). After 3 days of development, loose aggregates changed to tight aggregates, each of which was surrounded by a sheath. Cells located at the center seemed to be optically denser than peripheral cells (Figure $\mathbf{1 b}$, arrows). The optically denser part of an aggregate became a tight aggregate (a central cell mass) at 7 days of development (Figure 1c, an arrow). The peripheral cells surrounding the central masses were eventually lost, thus resulting in the formation of a macrocyst-like structure consisting of a central cell mass and a thick transparent wall (a macrocyst wall) surrounding it. Since the peripheral cells did not become transparent synchronously, three types of developmental forms eventually coexisted (Figures 1d-1f). As described above, the structure consisting of a central cell mass and peripheral cells surrounding it (Figure 1d), and the macrocyst-like structure consisting of a central cell mass and a thick transparent wall surrounding it (Figure 1e) were observed as the first and second forms. In addition, empty walls without central cell masses were sometimes observed as the third form (Figure 1f).

Although macrocyst-like structures are morphologically similar to normally formed macrocysts, it is presently unknown whether or not the central cell mass is a zygotic giant cell containing endocytes.

When the macrocyst-like structure was stained with Calcofluor before the completion of a transparent wall, the whole of the central cell mass was stained. As the outer surface of the peripheral cells surrounding the central cell mass was also stained, each peripheral cell was easily recognized. Comparing the size (diameter) of the cells, the cell shown by an arrow was about 4 times larger than that shown by an arrowhead (Figure 2a). Large-sized cells seem to fuse with each other to increase their size.

Some macrocyst-like structures were in process of forming transparent walls by losing peripheral cells. Since some lines consequently became visible in the macrocyst-like structure, it was realized that the central cell mass was surrounded by several parts of transparent zones (Figure $\mathbf{2 b}$ ).

Apparently, there was no significant difference in the macrocyst walls in either macrocyst-like structures or macrocysts. To confirm this similarity, the localization of GFP-ZYG1 fusion protein and cellulose in the macrocyst-like structure and macrocyst was examined by staining with Calcofluor. In the

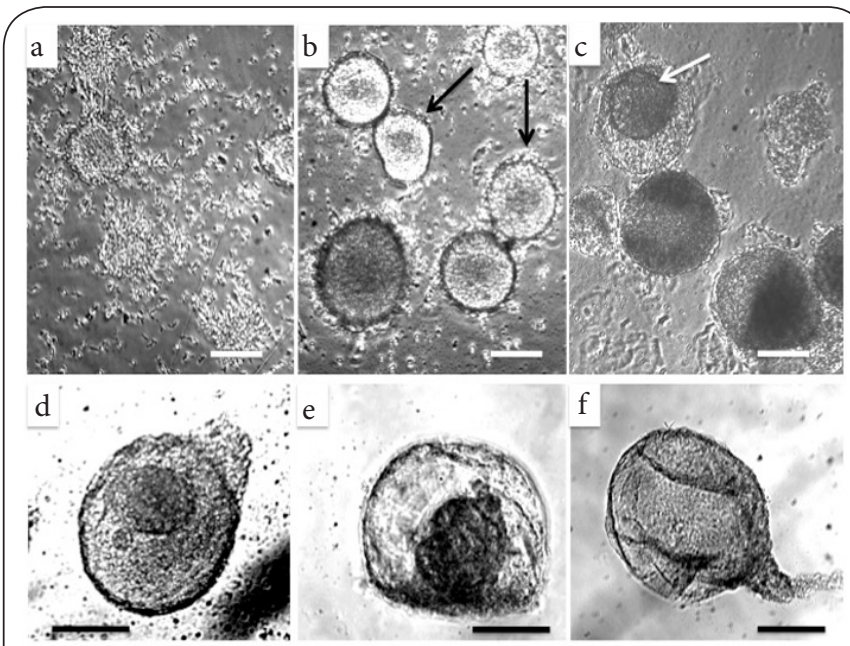

Figure 1. Development of GFP-ZYG1 ${ }^{\mathrm{OE}}$ cells. Starved GFP-ZYG1 ${ }^{\mathrm{OE}}$ cells were developed without their mating cells at a density of $6 \times 10^{6}$ cells/dish. (a) A phase-contrast photomicrograph at 1 day of development. Most of the cells finished aggregation and form loose aggregates. (b) A phase-contrast photomicrograph at 3 days of development. Loose aggregates change to tight aggregates, each of which is surrounded by a sheath (arrows). The cells located at the center of an aggregate seem to be optically denser than the peripheral cells. (c) A phase-contrast photomicrograph at 7 days of development. The optically denser part of an aggregate becomes a tight aggregate (a central cell mass, arrow). This structure looks like a macrocyst (a macrocyst-like structure). (d) A bright-field photomicrograph at 18 days of development. This shows a macrocyst-like structure consisting of a central cell mass and peripheral cells surrounding it. (e) A bright-field photomicrograph of a macrocyst-like structure at 17 days of development. This shows a complete macrocyst-like structure consisting of a central cell mass and a transparent macrocyst wall surrounding it. (f) A bright-field photomicrograph at 19 days of development. This shows a cell mass consisting of only transparent zones. Bars: $100 \mu \mathrm{m}$.

macrocyst-like structure, GFP-ZYG1 fusion protein was localized at the whole central cell mass and the outer layer of the macrocyst wall, co-localizing with Calcofluor staining (Figure 3a). In the macrocyst formed in GFPCONT cells co-cultured with V12M2 cells, GFP protein was localized at the whole giant cell and the outer layer of the macrocyst wall, co-localizing with Calcofluor staining (Figure $3 \mathbf{b}$ ). As a result, GFP-ZYG1 fusion protein and GFP protein were co-localized with cellulose in both macrocyst-like structures and macrocysts. This indicates the similarity of the macrocyst wall in both macrocyst-like structures and macrocysts. However, as GFPCONT protein also showed the same localization as GFP-ZYG1 fusion protein, co-localization of GFP-ZYG1 fusion protein with cellulose does not depend on ZYG1 overproduction.

When GFPCONT cells were developed without V12M2 cells, they formed loose aggregates, but never macrocyst-like structures. Therefore, the formation of macrocyst-like structures in GFPZYG1 ${ }^{\mathrm{OE}}$ cells was concluded to be due to ZYG1 overproduction. 


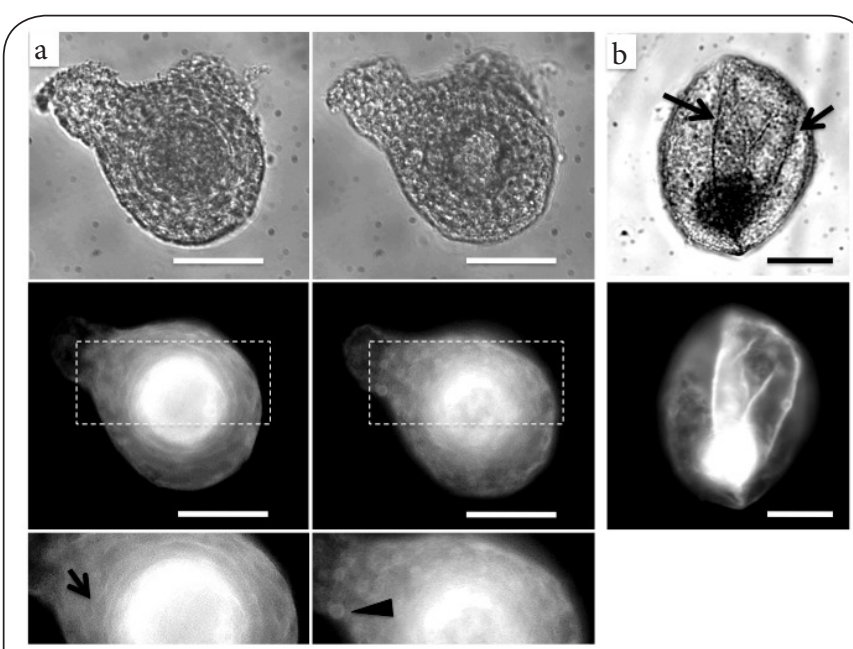

Figure 2. Peripheral cells stained with Calcofluor in a macrocyst-like structure. Starved GFP-ZYG1 ${ }^{\mathrm{OE}}$ cells were developed as described in the legend of Figure 1. After staining with Calcofluor, a macrocyst at 9 days of development was photographed. (a) The right hand panels show the same field as the left hand panels taken with slight focusing on the upper level. Upper panels: Bright-field photomicrographs. A cell aggregate consisting of a central cell mass surrounded by peripheral cells is shown. Middle panels: Fluorescence photomicrographs corresponding to the upper images. Lower panels: Magnified photographs designated by the dotted rectangular frames in the middle panels. Since the outer surface of the peripheral cells beside the central cell mass is stained with Calcofluor, each peripheral cell is easily recognized. Among them, large-sized cells (arrow) are observed, in addition to smaller cells (arrowhead). (b) Upper panel: A bright-field photomicrograph of a macrocystlike structure at 9 days of development. Zones containing peripheral cells become transparent, and some lines are visible (arrows). As somewhat membraneous lines are observed in the macrocyst wall, the macrocyst wall seems to consist of several parts of transparent zones (arrows). Lower panel: A fluorescence photomicrograph corresponding to the upper image. The macrocyst-like structure is in the process of forming transparent walls by losing peripheral cells, though some cells are still retained and do not disappear completely. Bars: $50 \mu \mathrm{m}$.

Macrocyst formation by GFP-ZYG1 ${ }^{\mathrm{OE}}$ and GFP ${ }^{\mathrm{CONT}}$ cells co-cultured with V12M2 cells

To elucidate the function of ZYG1 protein during macrocyst formation, GFP-ZYG $1{ }^{\mathrm{OE}}$ cells were developed with V12M2 cells. As a result, mainly two types of developmental forms were found: naturally-occurring mature macrocysts surrounded with a thick macrocyst wall (Figure 4Aa) and globular bodies (Figure 4Ab). These forms were also found when GFPCONT cells were developed with V12M2 cells as controls (Figure 4Ac). In consequence, there seemed to be no essential differences in the developmental forms between GFP-ZYG1 ${ }^{\mathrm{OE}}$ and GFPCONT cells. In macrocysts, two types of macrocyst wall were observed. One was lined with peripheral cells (Figure 4Aa, arrowed), and another with few peripheral cells (Figure 4Aa, arrowhead).

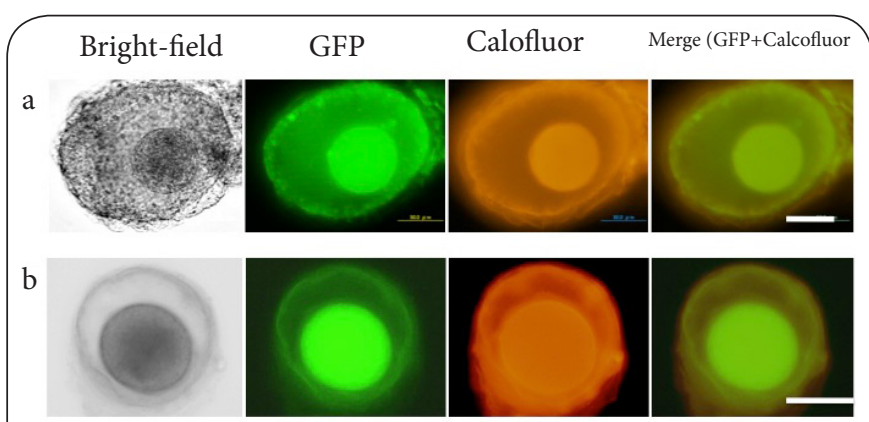

Figure 3. Localization of GFP-ZYG1 fusion protein, GFP protein and cellulose in the macrocyst-like structure and macrocyst. Bright-field photomicrographs and fluorescence photomicrographs of the same fields are shown. Calcofluor staining is shown in orange and GFP protein is shown in green. (a) Starved GFP-ZYG1 ${ }^{\mathrm{OE}}$ cells were developed as described in the legend of Figure 1. After staining with Calcofluor, a macrocyst-like structure at 25 days of development was photographed. GFP-ZYG1 fusion protein is localized at the whole central cell mass and the outer layer of the macrocyst wall, co-localizing with Calcofluor staining. (b) Starved $\mathrm{GFP}^{\mathrm{CONT}}$ and V12M2 cells were mixed at a ratio of $1: 1$ at a density of $6 \times 10^{6}$ cells/dish and developed. After staining with Calcofluor, a macrocyst at 22 days of development was photographed. GFP protein is localized at a whole giant cell and the outer layer of a macrocyst wall, co-localizing with Calcofluor staining. Bars: $50 \mu \mathrm{m}$.

Macrocysts formed by GFPCONT cells co-cultured with V12M2 cells also showed two types of macrocyst wall. Although most of the macrocyst walls were clear zones in mature macrocysts developed for 21 days (Figure 4Ad), macrocyst walls in which peripheral cells were still retained were sometimes observed even after 21 days of development (Figure 4Ae).

With regard to globular bodies, there has been no precise description yet. The mature globular bodies were filled with endocytes (Figure 4B, upper panel, arrowhead). Apparently, a thick macrocyst wall was not formed in the globular body. However, as the periphery of the globular bodies was stained with Calcofluor (Figure 4B, lower panel, arrowed), it is evident that they have a thin wall consisting of cellulose.

To precisely examine how globular bodies are formed, the developmental process in a mixed culture of GFP-ZYG $1^{\mathrm{OE}}$ and V12M2 was observed under phase-contrast microscopy and also recorded using time-lapsed imaging. Cell aggregates (arrowhead) and giant cells (arrows) were formed at 1 day of development after starvation, although many cells remained as non-aggregated single cells (Figure 5a). After 4 days of development, giant cells full of granular endocytes appeared (Figure $5 \mathbf{b}$, arrows). This was followed by formation of globular bodies after 6 days of development (Figure 5c, arrows). As the developmental process did not progress synchronously, giant cells and globular bodies coexisted for several days of development.

When GFPCONT cells were developed with V12M2 cells as controls, they showed a similar developmental process as 

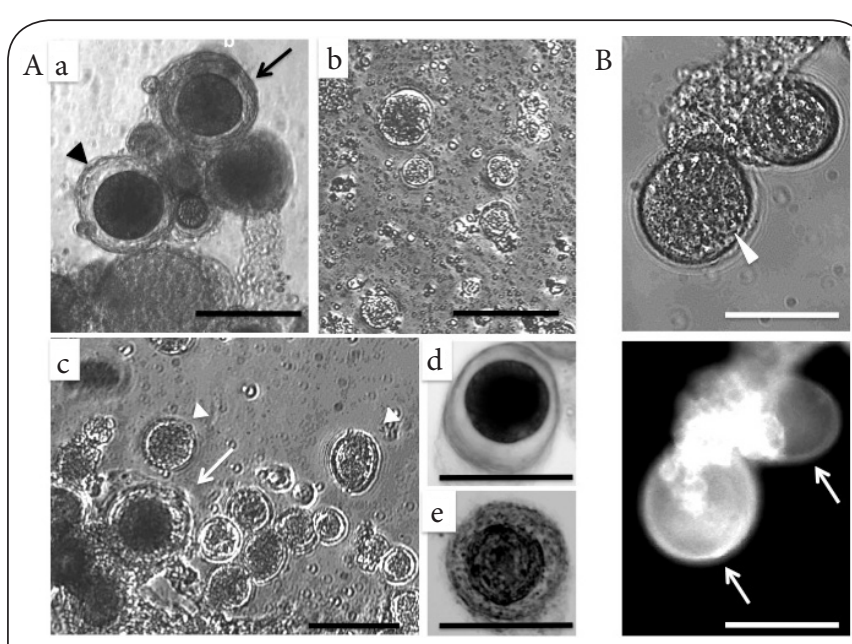

Figure 4. Formation of globular bodies. (A) Final structures formed by co-cultures of GFP-ZYG $1^{\mathrm{OE}}$ or GFP ${ }^{\mathrm{CONT}}$ cells with V12M2 cells. (a) and (b) Starved GFP-ZYG1 ${ }^{\mathrm{OE}}$ cells were mixed with V12M2 cells at a ratio of 1:1 at a density of $6 \times 10^{6}$ cells/dish and developed. (a) A bright-field photomicrograph of macrocysts with thick macrocyst walls at 5 days of development. Two kinds of macrocyst wall are observed: one is lined with peripheral cells (arrow), and another with few peripheral cells (arrowhead). (b) A phase-contrast photomicrograph of globular bodies at 6 days of development. (c-e) Starved GFPCONT cells were mixed withV12M2 cells at a ratio of 1:1 at a density of $6 \times 10^{6}$ cells/dish and developed. (c) A phase-contrast photomicrograph of a macrocyst surrounded with a macrocyst wall (arrow) and globular bodies (arrowheads) at 7 days of development. (d) A bright-field photomicrograph of a macrocyst with a clear macrocyst wall at 21 days of development. e A bright-field photomicrograph of a macrocyst with a macrocyst wall filled with cells at 21 days of development. Bars: $100 \mu \mathrm{m}$. (B) Formation of cellulosic walls in globular bodies. Starved GFP-ZYG1 ${ }^{\mathrm{OE}}$ and V12M2 cells were developed as described in A. At 12 days of development, globular bodies were photographed after staining with Calcofluor. Upper panel: A phase-contrast photomicrograph of globular bodies. They contain endocytes (arrowhead). Lower panel: a fluorescence photomicrograph corresponding to the upper panel. As the periphery of globular bodies is stained with Calcofluor (arrows), it is evident that the globular body is surrounded by a thin cellulosic wall. Bars: $50 \mu \mathrm{m}$.

shown in a mixed culture of GFP-ZYG $1^{\mathrm{OE}}$ and V12M2 cells. They were forming aggregates and giant cells (arrowed) at 1 day of development (Figure $\mathbf{5 d}$ ). After 5 days of development, giant cells full of granular endocytes appeared (Figure 5e, arrows). This was followed by the formation of globular bodies after 6 days of development (Figure 5f, arrows).

Time-lapse recording revealed that free-living giant cells ruffled their lamellipodia. As the free-living giant cells contained small particles, amoebae engulfed by them possibly changed to small particles (endocytes) [1]. It was sometimes observed that a giant cell itself was engulfed by another giant cell (Movie 1). The free-living giant cells containing endocytes suddenly stopped moving, retracted their lamellipodia and formed globular bodies (Figure 6 and Movie 2). Occasionally,

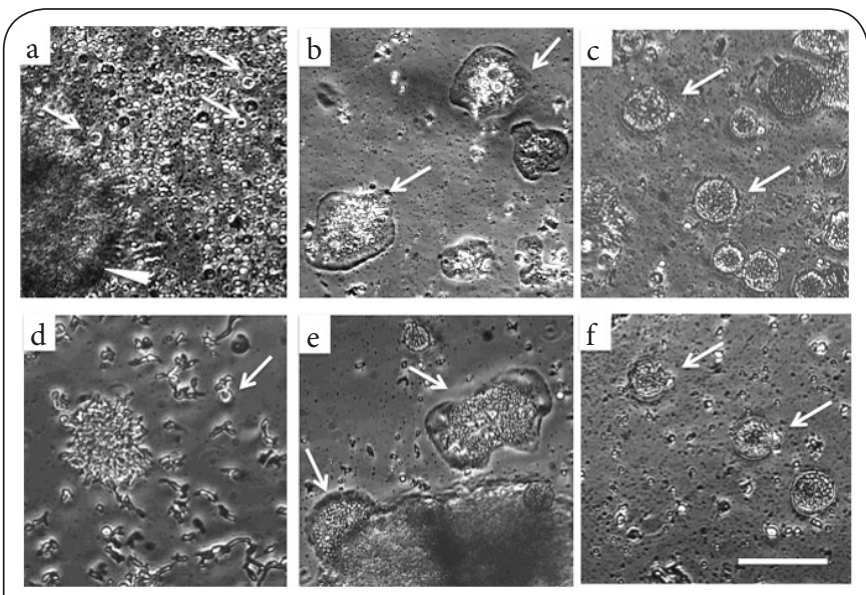

Figure 5. The developmental process of globular body formation by GFP-ZYG1 ${ }^{\mathrm{OE}}$ or GFP ${ }^{\mathrm{CONT}}$ cells co-cultured with V12M2 cells. a- c Starved GFP-ZYG1 ${ }^{\mathrm{OE}}$ and V12M2 cells were developed as described in the legend of Figure 4. (a) A phasecontrast photomicrograph at 1 day of development. Large aggregates (arrowhead) and free-living giant cells (arrows) are formed, though many cells remain as single cells. (b) A phasecontrast photomicrograph at 4 days of development. Giant cells filled with granular endocytes are formed (arrows). (c) A phase-contrast photomicrograph of globular bodies (arrows) at 6 days of development. d- f Starved GFPCONT and V12M2 cells were developed as described in the legend of Figure 4. (d) A phase-contrast photomicrograph at 1 day of development. Cells are forming an aggregate. A free-living giant cell (arrow) is observed. (e) A phase-contrast photomicrograph at 5 days of development. Giant cells filled with granular endocytes are formed (arrows). (f) A phase-contrast photomicrograph of globular bodies (arrows) at 6 days of development. Bar: 100 $\mu \mathrm{m}$.

globular bodies returned to the giant cells spreading their lamellipodia. In this case, however, they finally became globular bodies.

\section{Discussion}

In the present study, the developmental process and forms of GFP-ZYG1 ${ }^{\text {OE }}$ cells overproducing GFP-ZYG1 fusion protein were examined. As a result, the interesting findings concerning the function of ZYG1 and the developmental process of macrocyst formation were obtained.

When GFP-ZYG $1^{\mathrm{OE}}$ or GFPCONT cells were co-cultured with V12M2 cells, they formed macrocysts and many free-living giant cells. The free-living giant cells contained endocytes, retracted their lamellipodia and developed directly into globular bodies. Globular bodies have the characteristics of a macrocyst including the formation of giant cells containing endocytes and a wall stained with Calcofluor, though they do not form a thick macrocyst wall. Consequently, the globular body is likely the macrocyst formed directly by a giant cell without the process of cell aggregation.

Since globular bodies were also observed in a mixed culture of GFPCONT and V12M2 cells, the formation of the globular body 


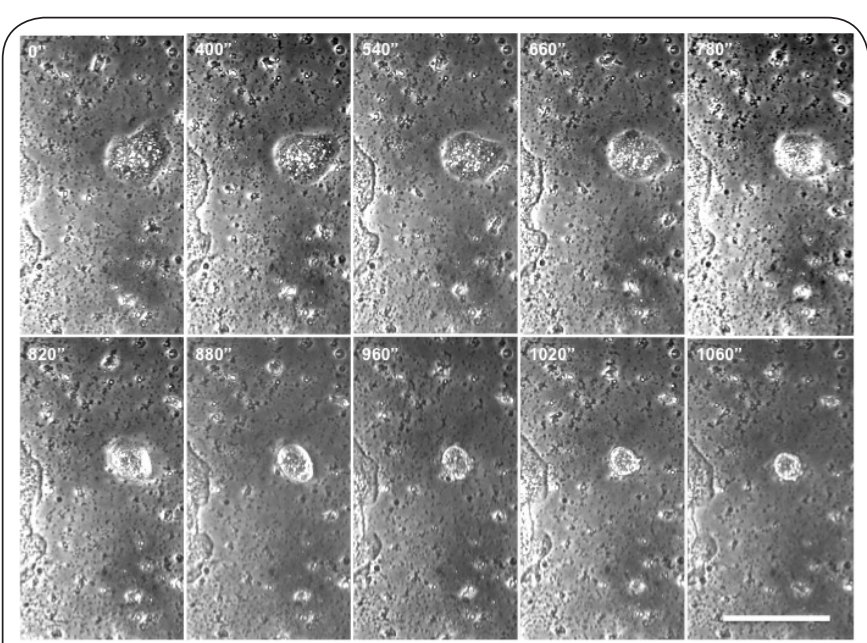

Figure 6. The time-lapse imaging of a globular body formation. Starved GFP-ZYG1 ${ }^{\mathrm{OE}}$ and V12M2 cells were developed as described in the legend of Figure 4. Time-lapse imaging was performed at 20 -second intervals. A free giant cell containing endocytes suddenly stops movement, retracts its lamellipodia and then forms a globular body. Bar: $100 \mu \mathrm{m}$.

would not be caused by ZYG1 overproduction.

When GFP-ZYG1 ${ }^{\text {OE }}$ cells were developed without V12M2 cells, they formed macrocyst-like structures. The macrocyst-like structure consisted of a central cell mass with a macrocyst wall surrounding it (Figure 1e). When GFPCONT cells were developed without V12M2 cells, they formed loose aggregates, but never macrocyst-like structures. Therefore, the formation of macrocyst-like structures is apparently due to ZYG1 overproduction. It is presently unknown whether the central cell mass is a zygotic giant cell containing endocytes. Considering that ZYG1 is known to induce cell fusion $[\mathbf{2 1 , 2 3 ]}$, it is supposed that globular bodies formed only by cell fusion may be formed by ZYG1 overproduction. However, the fact that the GFPZYG1 ${ }^{\mathrm{OE}}$ cells failed to form globular bodies without the help of mating type cells seemed to indicate that the functional zygotic giant cells might not be formed only by ZYG1 overproduction. On the other hand, it is most likely that the wall formation in a macrocyst-like structure may be induced by ZYG1 overproduction.

In the macrocyst-like structure, GFP-ZYG1 fusion protein, as well as cellulose, was localized at the whole central cell mass and the outer layer of the macrocyst wall (Figure 3a), as macrocysts formed by co-cultures of GFPCONT with V12M2 cells (Figure $3 \mathbf{b}$ ). This indicates the similarity in macrocyst walls of macrocyst-like structures and macrocysts. Consequently, according to the descriptions by Filosa and Dengler (1972), the thick transparent zone of the macrocyst-like structure corresponds to the thin inner layer and thick middle layer of the macrocyst wall in the macrocyst. The outer layer of the macrocyst-like structure, stained with Calcofluor, corresponds to the electron-dense, thin outer layer of the macrocyst wall in the macrocyst.
The formation process of transparent zones in macrocystlike structures was also suggested in the present study: that is, peripheral cells in the macrocyst-like structure increase their size by cell fusion and eventually become transparent zones (Figure 2). Coupling with the enlarged cells, GFP-ZYG1 fusion protein may be pushed toward the cell membrane and co-localized finally with cellulose at the outer layer of the macrocyst wall (Figure 3). Since ZYG1 is known to induce cell fusion [21], it is quite possible that ZYG1 may induce cell fusion in the early stages of macrocyst wall formation.

In macrocysts formed by GFP-ZYG1 ${ }^{\text {OE }}$ or GFPCONT cells COcultured with $\mathrm{V} 12 \mathrm{M} 2$ cells, two types of macrocyst wall were observed; a clear zone (Figure 4Aa, arrowhead and 4Ad) and a zone filled with cells (Figure 4Aa, arrowed and $4 A$ e). From this, it is supposed that the cell fusion process could be involved also in the wall formation of the macrocyst. The macrocyst wall is considered to be a product synthesized by a giant cell between the cell membrane and the outermost fibrillar sheath [1]. Both giant and periphery cells are possibly involved in the wall formation. However, at present, the accumulation of evidence is insufficient to clarify the precise mechanism of wall formation.

In the absence of a zyg 1 in GFPCONT cells, how is the macrocyst wall formed in the macrocysts? Since the existence of a $z y g 1$ homologue in V12M2 genome has been suggested by the genomic Southern analysis [21], ZYG1 could be supplied by V12M2 cells.

The idea of macrocyst wall formation by cell fusion of peripheral cells suggests that peripheral cells surrounding a giant cell might be necessary for the formation of transparent zones in a macrocyst wall. This may explain the reason why a globular body formed from a free-living giant cell has no thick transparent zones in a macrocyst wall. From the observations that the globular body is not surrounded by peripheral cells, cell fusion and subsequent vacuolization may not occur outside of a giant cell, thus resulting in lack of transparent zones of a macrocyst wall.

Recently, by BLAST search $[\mathbf{2 6 , 2 7 ]}$, uncharacterized ZYG1like protein has been reported from prokaryotes such as Enterobacter cloacae (DDBJ/EMBL/GenBank, accession number AYIG01000002, E value: 3e-93) and Escherichia coli (DDBJ/ EMBL/GenBank, accession number AWCH01000020, Evalue: 3e-91). As cellular slime molds except axenic strains such as Ax2, feed on bacteria phagocytotically as a food supply, their cells contain a lot of bacteria. It might be that a long time ago a bacterial zyg1 gene incorporated into the genome of cellular slime mold. Considering the functions of ZYG1, the information about cyst formation also might originate from bacteria. We hope that these interesting ideas will be further examined in the future.

\section{Competing interests}

The authors declare that they have no competing interests. 
Amagai et al. Research Journal of Developmental Biology 2014, http://www.hoajonline.com/journals/pdf/2055-4796-1-2.pdf

Authors' contributions

\begin{tabular}{|l|c|c|c|c|c|}
\hline Authors' contributions & AA & FT & TU & TA & YM \\
\hline Research concept and design & $\checkmark$ & -- & -- & -- & -- \\
\hline Collection and/or assembly of data & $\checkmark$ & $\checkmark$ & $\checkmark$ & $\checkmark$ & -- \\
\hline Data analysis and interpretation & $\checkmark$ & -- & -- & -- & $\checkmark$ \\
\hline Writing the article & $\checkmark$ & -- & -- & -- & -- \\
\hline Final approval of article & $\checkmark$ & -- & -- & -- & -- \\
\hline
\end{tabular}

\section{Acknowledgement}

We thank Dr. lan G. Gleadall, Tohoku University, and Dr. Richard Halberstadt for their critical reading and helpful comments regarding this manuscript.

\section{Publication history}

Senior Editor: Tzi Bun Ng, The Chinese University of Hong Kong, China.

Received: 18-Aug-2014 Final Revised: 17-Sep-2014

Accepted: 11-Oct-2014 Published: 17-Oct-2014

\section{References}

1. Filosa MF and Dengler RE. Ultrastructure of macrocyst formation in the cellular slime mold, Dictyostelium mucoroides: extensive phagocytosis of amoebae by a specialized cell. Dev Biol. 1972; 29:1-16. | Article | PubMed

2. Nickerson AW and Raper KB. Macrocysts in the life cycle of the Dictyosteliaceae. Germination of the macrocysts. Amer J Bot. 1973; 60:247-253. | Article

3. Filosa MF and Chan M. The isolation from soil of macrocyst-forming strains of the cellular slime mould Dictyostelium mucoroides. J Gen Microbiol. 1972; 207:491-495. | Article

4. Clark MA, Francis $D$ and Eisenberg R. Mating types in cellular slime molds. Biochem Biophys Res Commun. 1973; 52:672-8. | Article | PubMed

5. Erdos GW, Raper KB and Vogen LK. Mating Types and Macrocyst Formation in Dictyostelium discoideum. Proc Natl Acad Sci U S A. 1973; 70:1828-30. | Article | PubMed Abstract | PubMed Full Text

6. Erdos GW, Raper KB and Vogen LK. Sexuality in the cellular slime mold Dictyostelium giganteum. Proc Natl Acad Sci U S A. 1975; 72:970-3. | Article | PubMed Abstract | PubMed Full Text

7. O'Day DH. Aggregation during sexual development in Dictyostelium discoideum. Can J Microbiol. 1979; 25:1416-26. | Article | PubMed

8. O'Day DH and Durston AJ. Evidence for chemotaxis during sexual development in Dictyostelium discoideum. Can J Microbiol. 1979; 25:542-4. | PubMed

9. Amagai A. A novel function of ethylene. Gene Regul Syst Bio. 2009; 3:2130. | Article | PubMed Abstract | PubMed Full Text

10. Amagai A. Regulatory mechanism in sexual and asexual cycles of Dictyostelium. In Montanaro G and Dichio B (eds), Advances in Selected Plant Physiology Aspects. 2012; 327-344. | Book

11. Amagai A. Induction of zygote formation by ethylene during the sexual development of the cellular slime mold Dictyostelium mucoroides. Differentiation. 1989; 41:176-183. | Article

12. O'Day DH and Lydan MA. The regulation of membrane fusion during sexual development in Dictyostelium discoideum. Biochem Cell Biol. 1989; 67:321-6. I PubMed

13. Suzuki T, Amagai A and Maeda Y. Cyclic AMP and $\mathrm{Ca}^{2+}$ as regulators of zygote formation in the cellular slime mold Dictyostelium mucoroides. Differentiation. 1992; 49:127-32. | Article | PubMed

14. Chagla $\mathrm{AH}$, Lewis KE and $\mathrm{O}^{\prime} \mathrm{Day} \mathrm{DH}$. $\mathrm{Ca}^{2+}$ and cell fusion during sexual development in liquid cultures of Dictyostelium discoideum. Exp Cell Res. 1980; 126:501-5. | Article | PubMed

15. Szabo SP, O'Day DH and Chagla AH. Cell fusion, nuclear fusion, and zygote differentiation during sexual development of Dictyostelium discoideum. Dev Biol. 1982; 90:375-82. | Article | PubMed
16. Kawai $S$, Maeda $Y$ and Amagai A. Promotion of zygote formation by protein kinase inhibitors during the sexual development of Dictyostelium mucoroides. Dev Growth Differ. 1993; 35:601-607. | Article

17. Gunther KE, Ramkisson H, Lydan MA and O'Day DH. Fertilization in Dictyostelium: pharmacological analyses and the presence of a substrate protein suggest protein kinase $\mathrm{C}$ is essential for gamete fusion. Exp Cell Res. 1995; 220:325-331. | Article

18. Amagai A. Ethylene as a potent inducer of sexual development. Dev Growth Differ. 2011; 53:617-23. | Article | PubMed

19. Altschul SF, Gish W, Miller W, Myers EW and Lipman DJ. Basic local alignment search tool. J Mol Biol. 1990; 215:403-10. | Article | PubMed

20. Pearson WR. Rapid and sensitive sequence comparison with FASTP and FASTA. Methods Enzymol. 1990; 183:63-98. | Article | PubMed

21. Amagai A. Involvement of a novel gene, zyg1, in zygote formation of Dictyostelium mucoroides. J Muscle Res Cell Motil. 2002; 23:867-74. Article I PubMed

22. Amagai A, Soramoto SS, Saito SH and Maeda Y. Ethylene induces zygote formation through an enhanced expression of zyg1 in Dictyostelium mucoroides. Exp Cell Res. 2007; 313:2493-503. | Article | PubMed

23. Amagai $A$, MacWilliams $H$, Isono $T$, Omatsu-Kanbe $M$, Urano $S$, Yamamoto $\mathrm{K}$ and Maeda Y. PKC-mediated ZYG1 phosphorylation induces fusion of myoblasts as well as of Dictyostelium cells. Inter J Cell Biol. 2012; 2012: 11. | Article

24. Eichinger L, Pachebat JA, Glockner G, Rajandream MA, Sucgang R, Berriman $\mathrm{M}$, Song J, Olsen R, Szafranski $\mathrm{K}$ and $\mathrm{Xu} \mathrm{Q}$, et al., The genome of the social amoeba Dictyostelium discoideum. Nature. 2005; 435:4357. | Article | PubMed Abstract | PubMed Full Text

25. Bonner JT. Evidence for the formation of cell aggregates by chemotaxis in the development of the slime mold Dictyostelium discoideum. J Exp Zool. 1947; 106:1-26. | Article | PubMed

26. Altschul SF, Madden TL, Schaffer AA, Zhang J, Zhang Z, Miller W and Lipman DJ. Gapped BLAST and PSI-BLAST: a new generation of protein database search programs. Nucleic Acids Res. 1997; 25:3389-402. | Article | PubMed Abstract | PubMed Full Text

27. Altschul SF, Wootton JC, Gertz EM, Agarwala R, Morgulis A, Schaffer AA and Yu YK. Protein database searches using compositionally adjusted substitution matrices. FEBS J. 2005; 272:5101-9. | Article | PubMed Abstract $\mid \underline{\text { PubMed Full Text }}$

\section{Citation:}

Amagai A, Takahashi F, Usui T, Abe T and Maeda Y. Induction of macrocyst wall formation by ZYG1 in Dictyostelium discoideum. Res J Dev Biol. 2014; 1:2. http://dx.doi.org/10.7243/2055-4796-1-2 